\title{
Phantom dark energy with tachyonic instability: metric perturbations
}

\author{
A. Sergienko ${ }^{a, b}$, V. Rubakov ${ }^{b}$ \\ a Physics Department, Moscow State University \\ ${ }^{b}$ Institute for Nuclear Research of the Russian Academy of Sciences, \\ 60th October Anniversary Prospect, 7a, 117312 Moscow, Russia
}

\begin{abstract}
We study the behavior of metric perturbations in a recently proposed model of phantom dark energy with tachyonic instability at long wavelengths. We find that metric perturbations exponentially grow in time, starting from very small values determined by vacuum fluctuations, and may become sizeable at late times. This property may be of interest for phenomenology.
\end{abstract}

\section{Introduction}

Among models of dark energy (for reviews see, e.g., Refs. $[1,2,3,4,5,6,7]$ ), perhaps the most exotic ones are those exhibiting phantom equation of state, $p=w \rho$ with $w<-1$. Observational data strongly constrain this possibility $[8,9,10,11,12,13]$, but by no means rule it out, especially if the equation of state parameter $w$ varies in time (which is the case in many phantom models). For phantom matter, weak energy condition is violated, signaling instabilities and possibly other pathologies. Indeed, the simplest phantom models like those involving negative energy scalar field [14] have ghosts in the spectrum at arbitrarily high spatial momenta, and hence are plagued by catastrophic vacuum instability unless one introduces Lorentz-violating cutoff into the theory $[15,16]$.

This situation is not generic, however. First, phantom behavior may be mimicked in scalar-tensor and $f(R)$ gravities $[17,18,19]$ which need not have instabilities at all. Second, in theories with Lorentz-violating phantom fields, the violation of weak energy condition at 
cosmological distances does not imply dangerous instabilities at much shorter length scales. Indeed, phenomenologically acceptable models have been proposed [20, 21, 22, 23] that have precisely this property. In particular, the model of Refs. [22, 23] has tachyon in the spectrum of small perturbations about homogeneous Lorentz-violating phantom background, but the instabilities occur at low spatial momenta only, so that their time scale may be roughly comparable to (though somewhat smaller than) the present age of the Universe.

The latter property may well be generic to a subclass of phantom models, in which phantom instability is of tachyonic nature. So, it is of interest to understand, using the model of Refs. [22, 23] as a prototype, what observable consequences the long-wavelength tachyonic instabilities may have. As a step in this direction, we calculate in this paper the metric perturbations generated by the tachyon modes in this model. We find no surprise: like the tachyon modes themselves, metric perturbations exponentially grow in time, starting from very small values determined by the quantum physics of vacuum fluctuations. This may give rise to potentially observable effects, as the Universe rapidly becomes less homogeneous at late times.

\section{The model}

The model for dark energy we study in this paper is the Einstein gravity interacting with a vector field $B_{\mu}$ and a scalar field $\phi$. Its construction combines two ideas. One [24] is that in the absence of gauge invariance, a theory of a vector field may nevertheless have healthy spectrum, provided that the background vector field is non-trivial. The other [25] has to do with the fact that with vector field(s), one can construct generally covariant one-derivative terms in the Lagrangian, that dominate at low momenta and become negligible at high momenta. Hence, the Lagrangian for the vector and scalar fields is [22]

$L=-\frac{1}{2} \alpha(\xi) D_{\mu} B_{\nu} D^{\mu} B^{\nu}+\frac{1}{2} \beta(\xi) D_{\mu} B_{\nu} D^{\mu} B_{\lambda} \frac{B^{\nu} B^{\lambda}}{\Lambda^{2}}+\frac{1}{2} \partial_{\mu} \phi \partial^{\mu} \phi+\epsilon \partial_{\mu} \phi B^{\mu}+\frac{M^{2}}{2} B_{\mu} B^{\mu}-\frac{m^{2}}{2} \phi^{2}$,

where $\xi=B_{\mu} B^{\mu} / \Lambda^{2}, \Lambda$ is an UV cut-off scale, dimensionless parameters $\alpha$ and $\beta$ are functions of $\xi$, and $\epsilon$ is a positive constant of dimension of mass. Upon introducing the notation $B^{2}=B_{\mu} B^{\mu}$, this Lagrangian can be rewritten as follows,

$$
L=-\frac{\alpha}{2} D_{\mu} B_{\nu} D^{\mu} B^{\nu}+\frac{\alpha+\gamma}{8} \frac{\partial_{\mu}\left(B^{2}\right) \cdot \partial^{\mu}\left(B^{2}\right)}{B^{2}}+\frac{1}{2} \partial_{\mu} \phi \partial^{\mu} \phi+\epsilon \partial_{\mu} \phi B^{\mu}+\frac{M^{2}}{2} B^{2}-\frac{m^{2}}{2} \phi^{2}
$$

where $\gamma(\xi)=\frac{B^{2}}{\Lambda^{2}} \beta(\xi)-\alpha(\xi)$. To simplify formulas, in what follows we assume that $\alpha(\xi)=$ const, $\gamma(\xi)=$ const. The range of parameters in which the model exhibits phantom behavior is

$$
M \lesssim m \ll \epsilon
$$


and $\alpha \sim \gamma$. Spatially flat homogeneous background in this model is described by

$$
g_{\mu \nu}=a^{2}(\eta) \eta_{\mu \nu}, \quad B_{0}=a(\eta) X(\eta), \quad \phi=\varphi(\eta)
$$

where $\eta$ is conformal time. Importantly, temporal component of the vector field does not vanish, which ensures that the expression (1) makes sense, and that the spectrum of perturbations about this background is healthy [22, 23] at high spatial momenta (no ghosts, tachyons or superluminally propagating modes), provided that

$$
\alpha>\gamma>0
$$

As we will see shortly, one of the scalar modes of perturbations becomes a tachyon at sufficiently low momenta.

Cosmology in this model, with the usual and dark matter added, is fairly interesting [23]. In a wide range of initial data for $X$ and $\varphi$, these fields stay constant at early, matter dominated stage, and the corresponding equation of state is $w=-1$. Then they start evolving, the dark energy equation of state first being normal, $w>-1$, and later phantom, $w<-1$. The evolution often occurs in the slow roll regime, so that $|w+1|$ is small in the entire course of the cosmological expansion. Finally, the system approaches the de Sitter attractor, which to the leading order in $m / \epsilon, M / \epsilon$ is characterized by the following values of the Hubble parameter and fields,

$$
H_{A}=\frac{M}{\sqrt{3 \alpha}}, \quad X_{A}=-\frac{m M_{P L}}{\sqrt{12 \pi} \epsilon}, \quad \varphi_{A}=\frac{M M_{P L}}{\sqrt{4 \pi \alpha} m} .
$$

The interesting regime in which dark energy contributes substantially to the total energy density, occurs at

$$
H \sim H_{A}, \quad X \sim X_{A}, \quad \varphi \sim \varphi_{A}
$$

In what follows, we will be interested precisely in this range of the cosmological variables.

\section{Tachyonic perturbations}

The linearized perturbations of dark energy fields break up into vector and scalar parts. The vector sector is healthy at all spatial momenta, whereas the scalar sector has tachyons at relatively low momenta. Hence, we concentrate on the scalar sector. In Minkowski spacetime, the momenta at which one of the modes is tachyonic are of order $P \sim \epsilon$, and the corresponding "frequencies" are of the same order $[22,23]$. In the expanding Universe, a mode of a given conformal momentum $p$ becomes tachyonic as the physical momentum 
redshifts down to $P \equiv p / a \sim \epsilon$. Our purpose is to calculate the metric perturbations generated by the tachyonic modes ${ }^{1}$.

With scalar perturbations included, the fields and metric in conformal Newtonian gauge are given by

$$
\begin{gathered}
B_{0}(\eta, \mathbf{x})=a(\eta) X(\eta)+b_{0}(\eta, \mathbf{x}), \quad B_{i}(\eta, \mathbf{x})=b_{i}(\eta, \mathbf{x}), \quad \phi(\eta, \mathbf{x})=\varphi(\eta)+\frac{\chi(\eta, \mathbf{x})}{a(\eta)} \\
d s^{2}=a^{2}(\eta)\left[(1+2 \Phi) d \eta^{2}-(1-2 \Phi) d \mathbf{x}^{2}\right]
\end{gathered}
$$

where $b_{i}$ is a longitudinal vector, and we have made use of the fact that the linearized energy-momentum tensor of the fields $B^{\mu}$ and $\phi$ has zero anisotropic stress.

The complete expressions for the quadratic action and linear equations for perturbations in the slow roll regime (i.e., neglecting terms suppressed by $X^{\prime \prime}, X^{\prime}, \varphi^{\prime \prime}, \varphi^{\prime}$; hereafter prime denotes $\frac{\partial}{\partial \eta}$ ) are given in Appendix for completeness. However, we are interested in momenta $P \sim \epsilon$. According to the relations (2), modes of interest have relatively high spatial momenta and frequencies,

$$
P, \Omega \gg m, M, \quad P, \Omega \gg H
$$

where $\Omega$ is the physical frequency. Note that in the regime (3) one has $H \sim M$, so the second of these inequalities is a consequence of the first one. For momenta obeying (4), the equations simplify considerably:

$$
\begin{aligned}
\chi^{\prime \prime}-\Delta \chi+\epsilon a b_{0}^{\prime}-\epsilon a \partial_{i} b_{i}-2 \epsilon a^{2} X \Phi^{\prime} & =0 \\
\epsilon a \chi^{\prime}-\gamma\left(b_{0}^{\prime \prime}-\Delta b_{0}\right)+\gamma a X\left(\Phi^{\prime \prime}-\Delta \Phi\right) & =0 \\
\epsilon a \partial_{i} \chi+\alpha\left(b_{i}^{\prime \prime}-\Delta b_{i}\right)-2 \alpha a X \partial_{i} \Phi^{\prime} & =0 \\
-\frac{3 M_{P L}^{2}}{4 \pi} a\left(\Phi^{\prime \prime}-\Delta \Phi\right)-2 \epsilon a X \chi^{\prime}+\gamma X\left(b_{0}^{\prime \prime}-\Delta b_{0}\right)+2 \alpha X \partial_{i} b_{i}^{\prime} & =0
\end{aligned}
$$

We are now in a position to solve these equations in the WKB approximation by writing

$$
\left\{\chi, b_{0}, b_{i}, \Phi\right\} \propto \exp \left(i \int \omega d \eta-i \mathbf{p x}\right)
$$

with slowly varying amplitudes. Before doing that, we note that at $P, \Omega \sim \epsilon$, one estimates from eq. (8)

$$
\Phi \sim \frac{X}{M_{P l}^{2} a}\left\{\chi, b_{0}, b_{i}\right\} .
$$

Therefore, the last terms in eqs. (5), (6) and (7), describing back reaction of the gravitational potential on the field perturbations, are suppressed as compared to other terms by

\footnotetext{
${ }^{1}$ In fact, the behavior of modes in Minkowski space-time at $P \lesssim M$ is somewhat more complicated [23]. This region of momenta is not of interest for our purposes, as it corresponds to physical momenta, and hence frequencies, small compared to the Hubble parameter, see (3).
} 
$X^{2} / M_{P l}^{2} \sim m^{2} / \epsilon^{2}$. Neglecting these terms, we arrive at the system of equations for dark energy perturbations, which we write in the leading order of the WKB approximation,

$$
\begin{aligned}
\left(\omega^{2}-p^{2}\right) \chi-i \epsilon a \omega b_{0}+i \epsilon a p b_{L} & =0, \\
i \epsilon a \omega \chi+\gamma\left(\omega^{2}-p^{2}\right) b_{0} & =0 \\
i \epsilon a p \chi-\alpha\left(\omega^{2}-p^{2}\right) b_{L} & =0
\end{aligned}
$$

where $b_{L}=\left(p_{i} / p\right) b_{i}$. This is basically the same system as that obtained in Minkowski spacetime. There are three modes, two of which are normal at all momenta, while the third one is tachyonic at low momenta. In terms of physical frequency, the dispersion relation for the latter mode reads

$$
\Omega^{2} \equiv \frac{\omega^{2}}{a^{2}}=P^{2}+\frac{\epsilon^{2}}{2 \gamma}\left(1-\sqrt{1+\frac{4 \zeta \gamma^{2}}{\epsilon^{2}} P^{2}}\right)
$$

where

$$
\zeta=\frac{1}{\alpha}+\frac{1}{\gamma}
$$

As promised, the tachyonic regime occurs at

$$
P<P_{c} \equiv \frac{\epsilon}{\sqrt{\alpha}}
$$

The corresponding solution at $P>P_{c}$ is given by

$$
\begin{aligned}
\chi & =C \cdot \frac{i\left(\omega^{2}-p^{2}\right)}{\epsilon \omega} f(\eta) \mathrm{e}^{i \frac{\pi}{4}} \\
b_{0} & =C \cdot \frac{a}{\gamma} f(\eta) \mathrm{e}^{i \frac{\pi}{4}} \\
b_{L} & =-C \cdot \frac{a p}{\alpha \omega} f(\eta) \mathrm{e}^{i \frac{\pi}{4}}
\end{aligned}
$$

where $f(\eta)$ is a slowly varying function, $C$ is an overall constant and the phase factor is introduced for convenience.

The function $f(\eta)$ can be found by noting that with our approximations, the action for the field perturbations reads

$$
S=\int d \eta d \mathbf{x}\left[\frac{\alpha}{2}\left(\left(b_{i}^{\prime}\right)^{2}-\left(\partial_{i} b_{j}\right)^{2}\right)+\frac{\gamma}{2}\left(\left(b_{0}^{\prime}\right)^{2}-\left(\nabla b_{0}\right)^{2}\right)+\frac{1}{2}\left(\left(\chi^{\prime}\right)^{2}-(\nabla \chi)^{2}\right)+\epsilon a\left(\chi^{\prime} b_{0}-\partial_{i} \chi b_{i}\right)\right] .
$$

The corresponding energy functional is

$$
E=\int d \mathbf{x}\left[\frac{\alpha}{2}\left(\left(b_{i}^{\prime}\right)^{2}+\left(\partial_{i} b_{j}\right)^{2}\right)+\frac{\gamma}{2}\left(\left(b_{0}^{\prime}\right)^{2}+\left(\nabla b_{0}\right)^{2}\right)+\frac{1}{2}\left(\left(\chi^{\prime}\right)^{2}+(\nabla \chi)^{2}\right)+\epsilon a \partial_{i} \chi b_{i}\right] .
$$


In the WKB approximation, this energy is conserved. Making use of the expressions (10) and requiring that energy of this solution is conserved, one finds

$$
f(\eta)=\left|\frac{\omega}{2\left(\frac{\left(\omega^{2}-p^{2}\right)^{2}}{\epsilon^{2}}+\zeta a^{2} p^{2}\right)}\right|^{\frac{1}{2}}
$$

The same result can be obtained by considering the conservation of the Wronskian of the system (5), (6), (7), which, with our approximations, is

$$
W=-i\left(\chi^{*} \chi^{\prime}+\gamma b_{0}^{*} b_{0}^{\prime}+\alpha b_{L}^{*} b_{L}^{\prime}+\epsilon a \chi^{*} b_{0}-\text { c.c. }\right) \text {. }
$$

Now, to find the overall constant in (10) we quantize the system with the action (11) at early times and obtain

$$
C=\frac{A_{\mathbf{p}}^{+}}{(2 \pi)^{3 / 2}}
$$

where the creation and annihilation operators obey the standard commutational relation $\left[A_{\mathbf{p}}^{-}, A_{\mathbf{p}^{\prime}}^{+}\right]=\delta\left(\mathbf{p}-\mathbf{p}^{\prime}\right)$.

We are interested in the behavior of perturbations in the tachyonic regime. The exponentially growing part is found by the standard WKB continuation of the expressions (10), (12) and (13) "beyond the turning point". In this way we finally obtain in the tachyonic regime

$$
\begin{aligned}
\chi & =\int \frac{d \mathbf{p}}{(2 \pi)^{3 / 2}}\left(\frac{i\left(\omega^{2}-p^{2}\right)}{\epsilon \omega} f A_{\mathbf{p}}^{+} \mathrm{e}^{-i \mathbf{p x}}+\text { h.c. }\right) \mathrm{e}^{\int|\omega| d \eta}, \\
b_{0} & =\int \frac{d \mathbf{p}}{(2 \pi)^{3 / 2}}\left(\frac{a}{\gamma} f A_{\mathbf{p}}^{+} \mathrm{e}^{-i \mathbf{p x}}+\text { h.c. }\right) \mathrm{e}^{\int|\omega| d \eta}, \\
b_{i} & =\int \frac{d \mathbf{p}}{(2 \pi)^{3 / 2}}\left(-\frac{p_{i} a}{\alpha \omega} f A_{\mathbf{p}}^{+} \mathrm{e}^{-i \mathbf{p x}}+\text { h.c. }\right) \mathrm{e}^{\int|\omega| d \eta},
\end{aligned}
$$

where $f(\eta)$ is still given by $(12)$.

\section{Gravitational potentials}

The gravitational potential $\Phi$ is determined by eq. (8). After straightforward calculation we obtain that at $P>P_{c}$ the explicit expression is

$$
\Phi=-\sqrt{\frac{4 \pi}{27}} \frac{m}{M_{P L} \epsilon}\left(\frac{4 \gamma P^{2}}{\epsilon^{2}} \frac{1}{\sqrt{1+\frac{4 \zeta \gamma^{2}}{\epsilon^{2}} P^{2}}-1}-1\right) \cdot C \cdot f(\eta) \mathrm{e}^{i \frac{\pi}{4}}
$$


where $C$ and $f(\eta)$ are the same as in (10). Continuing into the tachyonic region, we obtain

$$
\Phi=-\sqrt{\frac{4 \pi}{27}} \frac{m}{M_{P L} \epsilon} \int \frac{d \mathbf{p}}{(2 \pi)^{3 / 2}}\left[\left(\frac{4 \gamma P^{2}}{\epsilon^{2}} \frac{1}{\sqrt{1+\frac{4 \zeta \gamma^{2}}{\epsilon^{2}} P^{2}}-1}-1\right) f A_{\mathbf{p}}^{+} \mathrm{e}^{-i \mathbf{p x}}+\text { h.c. }\right] \mathrm{e}^{\int|\omega| d \eta} .
$$

This expression gets simplified towards the end of the development of instability, i.e. at $P \ll \epsilon$ (but still $P \gg H \sim M$ ). In that case the dispersion relation (9) reads

$$
\omega^{2}=-\frac{\gamma}{\alpha} p^{2}
$$

so that

$$
f(\eta)=\frac{\gamma^{3 / 4} \alpha^{1 / 4}}{(\alpha+\gamma)^{1 / 2}} \frac{1}{\sqrt{2 p} a(\eta)}
$$

Hence

$$
\Phi=-\sqrt{\frac{4 \pi}{27}} \frac{m}{M_{P L} \epsilon} \frac{\gamma^{3 / 4} \alpha^{1 / 4}(\alpha-\gamma)}{(\alpha+\gamma)^{3 / 2}} \int \frac{d \mathbf{p}}{(2 \pi)^{3 / 2}} \frac{1}{\sqrt{2 p} a}\left(A_{\mathbf{p}}^{+} \mathrm{e}^{-i \mathbf{p x}}+\text { h.c. }\right) \mathrm{e}^{\int|\omega| d \eta} .
$$

This is Gaussian random field whose variance we write in terms of the physical momentum and frequency:

$$
\left\langle\Phi^{2}(\mathbf{x}, t)\right\rangle=\frac{1}{27 \pi} \frac{\gamma^{3 / 2} \alpha^{1 / 2}(\alpha-\gamma)^{2}}{(\alpha+\gamma)^{3}}\left(\frac{m}{M_{P L} \epsilon}\right)^{2} \int P d P \exp \left(2 \int_{t_{c}}^{t}|\Omega| d t\right)
$$

where the moment $t_{c}$ corresponds to the beginning of the tachyonic regime, i.e.,

$$
P\left(t_{c}\right) \equiv \frac{p}{a\left(t_{c}\right)}=P_{c}
$$

Note that the exponential growth factor is large but finite in the formal limit $t \rightarrow \infty$. As an example, for constant $H$, the total growth factor is

$$
\int_{t_{c}}^{\infty} \Omega(t) d t=\frac{1}{H} \int_{0}^{P_{c}} \Omega(P) \frac{d P}{P}=d(\alpha, \gamma) \cdot \frac{\epsilon}{H}
$$

where $d(\alpha, \gamma)$ is of order one (for example, $\left.d\left(1, \frac{1}{2}\right) \approx 0.499\right)$.

\section{Discussion}

Recalling the relations (2) and (3) one observes that the gravitational potentials grow from very small values, set by vacuum fluctuations at the low momentum scale $P_{c} \sim \epsilon$, but they eventually may become large. On the one hand, as discussed in Ref. [23], this gives rise to a 
constraint on the parameters of the model we discuss in this paper: by requiring that $\Phi \ll 1$ at the present time, one obtains

$$
\epsilon \lesssim \frac{1}{d(\alpha, \gamma)} H_{0} \ln \left(\frac{M_{P L}}{H_{0}}\right) \simeq 280 H_{0} \quad \text { for } \quad \alpha=1, \quad \gamma=0.5
$$

where $H_{0}$ is the present value of the Hubble parameter. So, the model may serve as a viable description of dark energy at the expense of extra fine tuning, over and beyond the usual fine tuning required to get the right value for cosmic acceleration. On the other hand, with even more fine tuning, the dark energy induced gravitational potentials may be sizeable (and

still acceptably small) today. A novel feature here is that the emergence of these potentials would be a fairly recent phenomenon: the largest values of the exponential factor in (14) are obtained at late times when $\Omega(t)$ changes slowly. Also, the amplitude of perturbations would be peaked at a certain momentum $P$ : for high momenta, the tachyonic regime has not yet started, while for low momenta the integral in the exponent is saturated at early times, and hence is small. These properties may be potentially observable.

The authors are indebted to O. Khovanskaya, M. Libanov and M. Sazhin for helpful discussions. This work is supported in part by Russian Foundation for Basic Research, grant 08-02-00473.

\section{Appendix. Complete action and equations for pertur- bations}

For completeness, we present here the complete expressions for the quadratic action and linear equations for the scalar perturbations, valid in the slow roll regime. We use the conformal Newtonian gauge and neglect the terms suppressed by $X^{\prime \prime}, X^{\prime}, \varphi^{\prime \prime}, \varphi^{\prime}$. The quadratic action is

$$
S^{(2)}=\int d \eta d \mathbf{x} a^{4} L^{(2)}
$$


where

$$
\begin{array}{r}
a^{4} L^{(2)}=-\frac{M_{P L}^{2}}{\pi}\left(-\frac{3}{8} a^{2} \Phi \Delta \Phi-\frac{3}{2} a^{3} H \Phi \Phi^{\prime}-\frac{3}{2} a a^{\prime \prime} \Phi^{2}-\frac{3}{8} a^{2}\left(\Phi^{\prime}\right)^{2}\right)- \\
-\frac{\alpha}{2}\left[-\left(b_{i}^{\prime}-a X \partial_{i} \Phi-a H b_{i}\right)^{2}-a^{2} H^{2} b_{i}^{2}+2 a H\left(\partial_{i} b_{0}-a X \partial_{i} \Phi\right) b_{i}\right]- \\
-\frac{\alpha}{2}\left[\left(\partial_{i} b_{j}\right)^{2}-2 a \partial_{i} b_{i}\left(H b_{0}-X \Phi^{\prime}\right)+3\left(-a H b_{0}+4 a^{\prime} X \Phi+a X \Phi^{\prime}\right)^{2}\right]- \\
-\alpha a^{\prime} X\left(\partial_{i} \Phi b_{i}-3 b_{0} \Phi^{\prime}-6 a^{\prime} X \Phi^{2}-6 a X \Phi \Phi^{\prime}\right)+ \\
+\frac{\gamma}{2}\left[\left(b_{0}^{\prime}-a H b_{0}-a X \Phi^{\prime}\right)^{2}-\left(\partial_{i} b_{0}-a X \partial_{i} \Phi\right)^{2}\right]+ \\
+\frac{1}{2}\left(\chi^{\prime}-\chi a H\right)^{2}-\frac{1}{2}\left(\partial_{i} \chi\right)^{2}+\epsilon a\left(\chi^{\prime}-\chi a H\right)\left(b_{0}-2 a X \Phi\right)-\epsilon a \partial_{i} \chi b_{i}+ \\
+\frac{M^{2} a^{2}}{2}\left(b_{0}^{2}-4 a X b_{0} \Phi+4 a^{2} X^{2} \Phi^{2}-b_{i}^{2}\right)-\frac{m^{2} a^{2}}{2} \chi^{2} .
\end{array}
$$

From here we obtain the equations for the field perturbations:

$$
\begin{aligned}
& \chi^{\prime \prime}-\Delta \chi-\frac{a^{\prime \prime}}{a} \chi+m^{2} a^{2} \chi+\epsilon a b_{0}^{\prime}+2 \epsilon a^{\prime} b_{0}-\epsilon a \partial_{i} b_{i}-2 \epsilon a^{2} X \Phi^{\prime}-6 \epsilon a a^{\prime} X \Phi=0 \\
& \epsilon\left(a \chi^{\prime}-a^{\prime} \chi\right)-\gamma\left(b_{0}^{\prime \prime}-\Delta b_{0}-\frac{a^{\prime \prime}}{a} b_{0}\right)+\left(M^{2}-3 \alpha H^{2}\right) a^{2} b_{0}+2 \alpha a H \partial_{i} b_{i}+ \\
& +\gamma a X\left(\Phi^{\prime \prime}-\Delta \Phi\right)+2(3 \alpha+\gamma) a^{\prime} X \Phi^{\prime}+2\left(6 \alpha H^{2}-M^{2}\right) a^{3} X \Phi=0 \\
& \epsilon a \partial_{i} \chi+2 \alpha a H \partial_{i} b_{0}+\alpha\left(b_{i}^{\prime \prime}-\Delta b_{i}-\frac{a^{\prime \prime}}{a} b_{i}\right)+\left(M^{2}-\alpha H^{2}\right) a^{2} b_{i}-2 \alpha a X \partial_{i} \Phi^{\prime}-2 \alpha a^{2} H X \partial_{i} \Phi=0 \\
& -\frac{3 M_{P L}^{2}}{4 \pi}\left[a^{2}\left(\Phi^{\prime \prime}-\Delta \Phi\right)+2 a a^{\prime} \Phi^{\prime}-2\left(a a^{\prime \prime}-\left(a^{\prime}\right)^{2}\right) \Phi\right]-2 \epsilon a X\left(a \chi^{\prime}-a^{\prime} \chi\right)+\gamma a X\left(b_{0}^{\prime \prime}-\Delta b_{0}\right)- \\
& -6 \alpha a^{2} H X b_{0}^{\prime}-\gamma a^{\prime \prime} X b_{0}-2 M^{2} a^{3} X b_{0}+12 \alpha a^{3} H^{2} X b_{0}-6 \alpha a^{\prime \prime} X b_{0}+2 \alpha a X \partial_{i} b_{i}^{\prime}+ \\
& +a^{2} X^{2}(3 \alpha-\gamma) \Phi^{\prime \prime}+(\gamma-\alpha) a^{2} X^{2} \Delta \Phi+2(3 \alpha-\gamma) a^{3} H X^{2} \Phi^{\prime}+6 \alpha\left(a a^{\prime \prime}-5\left(a^{\prime}\right)^{2}\right) X^{2} \Phi+ \\
& +4 M^{2} a^{4} X^{2} \Phi=0
\end{aligned}
$$

Equations (5), (6), (7) and (8) follow from the latter equations in the regime (4), while the action (15) reduces to (11) in this regime.

\section{References}

[1] V. Sahni and A. A. Starobinsky, Int. J. Mod. Phys. D 9, 373 (2000) [arXiv:astroph/9904398]. 
[2] S. M. Carroll, Living Rev. Rel. 4, 1 (2001) [arXiv:astro-ph/0004075].

[3] T. Padmanabhan, Phys. Rept. 380, 235 (2003) [arXiv:hep-th/0212290].

[4] P. J. E. Peebles and B. Ratra, Rev. Mod. Phys. 75, 559 (2003) [arXiv:astro-ph/0207347].

[5] V. Sahni and A. Starobinsky, Int. J. Mod. Phys. D 15, 2105 (2006) [arXiv:astro$\mathrm{ph} / 0610026]$.

[6] S. Nojiri and S. D. Odintsov, Int. J. Geom. Meth. Mod. Phys. 4, 115 (2007) [arXiv:hepth/0601213].

[7] E. J. Copeland, M. Sami and S. Tsujikawa, Int. J. Mod. Phys. D 15, 1753 (2006) [arXiv:hep-th/0603057].

[8] P. Astier et al. [The SNLS Collaboration], Astron. Astrophys. 447, 31 (2006) [arXiv:astro-ph/0510447].

[9] A. G. Riess et al., arXiv:astro-ph/0611572.

[10] W. M. Wood-Vasey et al. [ESSENCE Collaboration], Astrophys. J. 666, 694 (2007) [arXiv:astro-ph/0701041].

[11] S. Cole et al. [The 2dFGRS Collaboration], Mon. Not. Roy. Astron. Soc. 362, 505 (2005) [arXiv:astro-ph/0501174].

[12] M. Tegmark et al. [SDSS Collaboration], Phys. Rev. D 74, 123507 (2006) [arXiv:astro$\mathrm{ph} / 0608632]$.

[13] E. Komatsu et al. [WMAP Collaboration], "Five-Year Wilkinson Microwave Anisotropy Probe (WMAP) Observations:Cosmological Interpretation," arXiv:0803.0547 [astro-ph].

[14] R. R. Caldwell, Phys. Lett. B 545, 23 (2002) [arXiv:astro-ph/9908168].

[15] S. M. Carroll, M. Hoffman and M. Trodden, Phys. Rev. D 68, 023509 (2003) [arXiv:astro-ph/0301273].

[16] J. M. Cline, S. Jeon and G. D. Moore, Phys. Rev. D 70, 043543 (2004) [arXiv:hep$\mathrm{ph} / 0311312]$.

[17] B. Boisseau, G. Esposito-Farese, D. Polarski and A. A. Starobinsky, Phys. Rev. Lett. 85, 2236 (2000) [arXiv:gr-qc/0001066].

[18] R. Gannouji, D. Polarski, A. Ranquet and A. A. Starobinsky, JCAP 0609, 016 (2006) [arXiv:astro-ph/0606287]. 
[19] L. Amendola, R. Gannouji, D. Polarski and S. Tsujikawa, Phys. Rev. D 75, 083504 (2007) [arXiv:gr-qc/0612180].

[20] L. Senatore, Phys. Rev. D 71, 043512 (2005) [arXiv:astro-ph/0406187].

[21] P. Creminelli, M. A. Luty, A. Nicolis and L. Senatore, JHEP 0612, 080 (2006) [arXiv:hep-th/0606090].

[22] V. A. Rubakov, Theor. Math. Phys. 149, 1651 (2006) [Teor. Mat. Fiz. 149, 409 (2006)] [arXiv:hep-th/0604153].

[23] M. Libanov, V. Rubakov, E. Papantonopoulos, M. Sami and S. Tsujikawa, JCAP 0708, 010 (2007) [arXiv:0704.1848 [hep-th]].

[24] B. M. Gripaios, JHEP 0410, 069 (2004) [arXiv:hep-th/0408127].

[25] M. V. Libanov and V. A. Rubakov, JHEP 0508, 001 (2005) [arXiv:hep-th/0505231]. 